\title{
Spatial Risk Measures and their Local Specification: \\ The Locally Law- Invariant Case
}

by

Hans Föllmer

\begin{abstract}
We consider convex risk measures in a spatial setting, where the outcome of a financial position depends on the states at different nodes of a network. In analogy to the theory of Gibbs measures in Statistical Mechanics, we discuss the local specification of a global risk measure in terms of conditional local risk measures for the single nodes of the network, given their environment. Under a condition of local law invariance, we show that a consistent local specification must be of entropic form. Even in that case, a global risk measure may not be uniquely determined by the local specification, and this can be seen as a source of "systemic risk", in analogy to the appearance of phase transitions in the theory of Gibbs measures.
\end{abstract}

\section{Introduction}

Consider a large network of financial institutions. At each node of the network we may want to assess the risk of the financial position taken at that node. This is usually done in terms of some monetary risk measure, which specifies the additional capital that is needed to make the position acceptable from the point of view of a supervising agency. Typically, such a local risk assessment is based on the marginal distribution which governs the behavior of the position at the given node. This way, however, we would neglect interactive effects not captured by the family of marginal distributions. It is therefore plausible to take a conditional approach such that the risk measure applied at a given node takes into account the situation at the other nodes of the network. In that case, the question arises whether these local conditional risk assessments can be aggregated in a consistent manner.

In this paper, our purpose is to explore, from a purely mathematical point view, the issue of consistent aggregation for conditional risk measures in a spatial setting. Let $I$ be a countable set of nodes, or sites, whose states vary in some state space $S$. Suppose that at each site $i \in I$ we are given a conditional convex risk measure $\rho_{\{i\}}$ which depends on the environment of $i$, described by the states of the other sites $j \neq i$. The question of consistent aggregation will be made precise by considering the following three problems:

(I) A problem of local consistency: How arbitrarily can we prescribe the conditional risk measures $\rho_{\{i\}}$ for the single sites $i \in I$ so that they can be aggregated, at least locally, in a consistent manner? More precisely, can the family $\left(\rho_{\{i\}}\right)$ be embedded in a family $\left(\rho_{V}\right)$ indexed by the finite subsets of $I$ such that each $\rho_{V}$ is a conditional convex risk measure depending on the environment of $V$, and the consistency condition $\rho_{W}\left(-\rho_{V}\right)=\rho_{W}$ is satisfied for any two finite subsets $V$ and $W$ such that $V \subseteq W$ ? If that is the case, then the family $\left(\rho_{V}\right)$ will be called a local specification of a convex risk measure.

(II) An existence problem: Is there a global convex risk measure $\rho$ that is consistent with the local specification $\left(\rho_{V}\right)$, that is, $\rho\left(-\rho_{V}\right)=\rho$ for any $V$ ?

(III) A uniqueness and a representation problem: Is such a global risk measure uniquely determined by the local specification $\left(\rho_{V}\right)$ ? And if this is not the case, how can we describe the structure of the set of all such global risk measures?

These three problems can be seen as non-linear analogues to three basic problems appearing in the theory of Gibbs measures in Statistical Mechanics. In the probabilistic approach to phase transitions initiated by R. L. Dobrushin [D], global probability measures on a space of spatial configurations, also called random fields, are specified by a family $\left(\pi_{V}\right)$ of local conditional probability distributions. Local consistency of such a local 
specification can be characterized in terms of interaction potentials, and therefore the corresponding random fields are also called Gibbs measures. Non-uniqueness of the global Gibbs measure is usually interpreted as the appearance of a phase transition. In our non-linear setting of convex risk measures, non-uniqueness of the global risk measure can be seen as one specific aspect of the much broader issue of systemic risk.

At the general level described so far, all three problems are to a large extent still open; some partial results on problems (II) and (III) will be discussed in $[\mathrm{CFK}]$. In the present paper we focus on the special case of local law-invariance, and here we can give explicit answers to all three problems. Local law-invariance will mean that the local conditional risk measures $\rho_{V}$ are based on the conditional probability distributions $\pi_{V}$ given by the local specification of a Gibbs measure. Under this condition, we are going to show that the local specification of a convex risk measure must be entropic with some parameter of risk aversion that may depend on the spatial tail field. This result is stated in Theorem 4.5, and it solves the problem of local consistency (I), as explained in Corollary 4.6. We also show that there is a positive answer to the existence problem (II), if the same is true for the underlying local specification $\left(\pi_{V}\right)$ of a Gibbs measure. The solution of Problem (III) is given by Theorem 4.7, which describes the structure of the class of all global convex risk measures consistent with the local specification $\left(\rho_{V}\right)$. This involves an extension of $\left(\rho_{V}\right)$ to a conditional entropic risk measure with respect to the spatial tail-field. Here we use an integral representation for the class of Gibbs measures coupled to the tail field which was obtained in $[\mathrm{F}]$, in analogy to E. B. Dynkin's construction of entrance boundaries for Markov processes in [Dy] and [Dy1]. In particular, the representation in Theorem 4.7 implies that uniqueness of the global risk measure holds if and only if the Gibbs measure is unique and the parameter of risk aversion is constant. In other words, there are two possible sources of non-uniqueness of the global risk measure: on the one hand a probabilistic phase transition and, on the other hand, a non-trivial tail-field dependence of the risk parameter. This is made precise in Corollaries 4.8 and 4.9 .

The entropic form of the local specification $\left(\rho_{V}\right)$ follows from a general result concerning the combined effect of consistency and law-invariance, which is of independent interest: Under mild regularity conditions, a law-invariant convex risk measure on a space $L^{\infty}(\Omega, \mathcal{F}, P)$ is entropic as soon as it is consistent with respect to just one sub- $\sigma$-field $\mathcal{F}_{0}$ that is properly contained in $\mathcal{F}$. The precise statement is given in Theorem 2.4. It can be seen as both an extension and a simplification of the result in $[\mathrm{KS}]$ that law-invariant dynamic risk measures are entropic. Our proof of Theorem 2.4 is inspired by the discussion of quasi-convex risk measures in $[\mathrm{CMMM}]$, in particular by the characterization of certainty equivalents within the class of law-invariant quasi-convex risk measures in their Lemma 11. But our consistency assumption is weaker, and this requires a conditional refinement of the key argument in [CMMM].

The paper is organized as follows. In Section 1 we recall some basic notions from the theory of convex risk measures, and in Section 2 we prove the general Theorem 2.4. In Section 3 we introduce our spatial setting. First we fix a local specification $\left(\pi_{V}\right)$ of a Gibbs measure, and we review the corresponding integral representation of Gibbs measures in terms of the spatial tail field. Then we define the local specification $\left(\rho_{V}\right)$ of a convex risk measure, and we assume that it is connected to $\left(\pi_{V}\right)$ by a condition of local law invariance. In Section 4, a local application of Theorem 2.4 provides the key to the entropic description of the local specification $\left(\rho_{V}\right)$ in Theorem 4.5. The next step consists in extending the local specification $\left(\rho_{V}\right)$ to a conditional entropic risk measure $\rho_{\infty}$ with respect to the spatial tail field. This will yield our final representation results in Theorem 4.7 and its corollaries.

\section{Preliminaries on risk measures and risk kernels}

In this section we recall some basic notions from the theory of monetary risk measures initiated in $[\mathrm{ADEH}],[\mathrm{H}],[\mathrm{FRG}]$, and [FS]. For more details see, for example, [FS1] and [FK].

For a measurable space $(\Omega, \mathcal{F})$ we denote by $M:=M_{b}(\Omega, \mathcal{F})$ the Banach space of all bounded measurable functions on $(\Omega, \mathcal{F})$. A real-valued functional $\rho$ on $M$ will be called a monetary risk measure if it is monotone, i.e., $\rho(X) \geq \rho(Y)$ whenever $X \leq Y$, cash-invariant, i.e., $\rho(X+m)=\rho(X)-m$ for constants $m$, and normalized, i.e., $\rho(0)=0$. If a monetary risk measure $\rho$ is also convex on $M$, then $\rho$ will be called a 
convex risk measure. A convex risk measure is called coherent if it is also positively homogeneous, that is, $\rho(\lambda X)=\lambda \rho(X)$ for any positive constant $\lambda$.

Now let $P$ be a probability measure on $(\Omega, \mathcal{F})$. If $\rho$ is a monetary risk measure on $M$ such that $\rho(X)=\rho(Y)$ whenever $X=Y P$-almost surely, then $\rho$ can also be considered as a monetary risk measure on the Banach space $L^{\infty}:=L^{\infty}(\Omega, \mathcal{F}, P)$. Such a risk measure is called law-invariant (with respect to $P$ ) if $\rho(X)=\rho(Y)$ whenever $X$ and $Y$ have the same distribution under $P$.

As an example, consider the entropic risk measure $e_{\beta}$ with parameter $\beta \in[0, \infty)$, defined by

$$
e_{\beta}(X)=\frac{1}{\beta} \log E_{P}\left[e^{-\beta X}\right] ;
$$

for $\beta=0$, this will be interpreted as the limiting linear case

$$
e_{0}(\beta):=\lim _{\beta \downarrow 0} e_{\beta}(X)=E_{P}[-X] .
$$

An entropic risk measure is clearly convex and law-invariant. Moreover, it has the following two regularity properties with respect to $P$ :

Definition 1.1. A monetary risk measure on $L^{\infty}$ is called strictly sensitive (with respect to $P$ ), if

$$
X \geq Y \quad P-\text { a.s., } \quad \rho(X)=\rho(Y) \quad \Longrightarrow \quad X=Y \quad P \text {-a.s.. }
$$

It is said to have the Lebesgue property (with respect to $P$ ) if

$$
\rho(X)=\lim _{n \rightarrow \infty} \rho\left(X_{n}\right)
$$

for any uniformly bounded sequence $\left(X_{n}\right)_{n=1,2, \ldots}$ in $L^{\infty}$ such that $X_{n} \rightarrow X$ P-a.s.

In Section 2 we are going to see that, conversely, any law-invariant convex risk measure on $L^{\infty}$ with these two properties is entropic as soon as it has an additional consistency property. This will involve the following notion of a conditional monetary risk measure.

Consider a $\sigma$-field $\mathcal{F}_{0} \subseteq \mathcal{F}$, and let us use the notation $L_{0}^{\infty}:=L^{\infty}\left(\Omega, \mathcal{F}_{0}, P\right)$.

Definition 1.2 A map $\rho_{0}$ from $L^{\infty}$ to $L_{0}^{\infty}$ is called a conditional monetary risk measure with respect to $\mathcal{F}_{0}$ if it satisfies the following three properties for any $X, Y \in L^{\infty}$ :

i) Monotonicity: $\rho_{0}(X) \geq \rho_{0}(Y) P$-a.s. whenever $X \leq Y$-a.s.

ii) Conditional cash invariance: for all $m \in L_{0}^{\infty}, \rho_{0}(X+m)=\rho_{0}(X)-m P$-a.s.

iii) Normalization: $\rho_{0}(0)=0$ P-a.s.

It is easy to see that any conditional monetary with respect to $\mathcal{F}_{0}$ has the following local property: when $X, Y \in L^{\infty}$ and $A_{0} \in \mathcal{F}_{0}$, then

$$
\rho_{0}\left(I_{A_{0}} X+I_{A_{0}^{c}} Y\right)=I_{A_{0}} \rho_{0}(X)+I_{A_{0}^{c}} \rho_{0}(Y) \quad P \text { - a.s.; }
$$

see, for example, [DS] or [FS1], Exercise 11.1.1

Definition 1.3. A monetary risk measure $\rho$ on $L^{\infty}$ is called $\mathcal{F}_{0}$-consistent if there exists a conditional monetary risk measure $\rho_{0}$ with respect to $\mathcal{F}_{0}$ such that

$$
\rho=\rho\left(-\rho_{0}\right),
$$

that is, $\rho(X)=\rho\left(-\rho_{0}(X)\right)$ for any $X \in L^{\infty}$. 
Remark 1.4. Note that $\mathcal{F}_{0}$-consistency is always satisfied in the two extreme cases $\mathcal{F}_{0}=\mathcal{F}$ mod $P$ (with $\left.\rho_{0}(X):=-X\right)$ and $\mathcal{F}_{0}=\{\emptyset, \Omega\} \bmod P\left(\right.$ with $\left.\rho_{0}(X):=\rho(X)\right)$.

Remark 1.5. An entropic risk measure $e_{\beta}$ is $\mathcal{F}_{0}$-consistent for every sub- $\sigma$-field $\mathcal{F}_{0} \subseteq \mathcal{F}$. Indeed, a direct computation shows that

$$
e_{\beta}(X)=e_{\beta}\left(-e_{\beta, 0}(X)\right)
$$

for any $X \in L^{\infty}$, where $e_{\beta, 0}$ denotes the conditional entropic risk measure with the same parameter $\beta$, defined by

$$
e_{\beta, 0}(X)=\frac{1}{\beta} \log E_{P}\left[e^{-\beta X} \mid \mathcal{F}_{0}\right] ;
$$

for $\beta=0$, this should be read as

$$
e_{0,0}(X):=\lim _{\beta \downarrow 0} e_{\beta, 0}(X)=E_{P}\left[-X \mid \mathcal{F}_{0}\right],
$$

in analogy to (1.2). In Section 2 we will show that, conversely, any law-invariant convex risk measure with the above properties is entropic as soon as it is $\mathcal{F}_{0}$-consistent for just one $\sigma$-field $\mathcal{F}_{0}$ that is "properly contained" in $\mathcal{F}$.

In the preceding definitions we have referred to a given probability measure $P$ on $(\Omega, \mathcal{F})$, and this will be the setting in Section 2. But for our discussion of locally specified spatial risk measures in Sections 3 and 4, it will be important to argue without such a global reference measure. For this reason we introduce the modified notions of a conditional risk measure in Definitions 1.6 and 1.7 below.

Let us first recall the classical definition of a stochastic kernel $\pi\left(\omega_{0}, d \omega_{1}\right)$ from a measurable space $\left(\Omega_{0}, \mathcal{F}_{0}\right)$ to a measurable space $\left(\Omega_{1}, \mathcal{F}_{1}\right)$ : For any $\omega_{0} \in \Omega_{0}, \pi\left(\omega_{o}, \cdot\right)$ is a probability measure on $\left(\Omega_{1}, \mathcal{F}_{1}\right)$, and for any $A_{1} \in \mathcal{F}_{1}$, the function $\pi\left(\cdot, A_{1}\right)$ on $\Omega_{0}$ is $\mathcal{F}_{0}$-measurable. For a probability measure $P$ on $\left(\Omega_{0}, \mathcal{F}_{0}\right)$ we denote by $P \pi$ the probability measure on $\left(\Omega_{1}, \mathcal{F}_{1}\right)$ defined by $P \pi\left[A_{1}\right]=\int \pi\left(\omega_{0}, A_{1}\right) P\left(d \omega_{0}\right)$. For two kernels $\pi_{i}$ from $\left(\Omega_{i}, \mathcal{F}_{i}\right)$ to $\left(\Omega_{i+1}, \mathcal{F}_{i+1}\right)(i=0,1)$, their composition $\pi_{0} \pi_{1}$ is defined as the stochastic kernel from $\left(\Omega_{0}, \mathcal{F}_{0}\right)$ to $\left(\Omega_{2}, \mathcal{F}_{2}\right)$ given by $\pi_{0} \pi_{1}\left(\omega_{0}, A_{2}\right)=\int \pi_{1}\left(\omega_{1}, A_{2}\right) d \pi_{0}\left(\omega_{0}, d \omega_{1}\right)$.

For a measurable space $(\Omega, \mathcal{F})$ and a sub- $\sigma$-field $\mathcal{F}_{0} \subseteq \mathcal{F}$, a regular conditional distribution on $(\Omega, \mathcal{F})$ with respect to $\mathcal{F}_{0}$ is defined as a stochastic kernel $\pi$ from $\left(\Omega, \mathcal{F}_{0}\right)$ to $(\Omega, \mathcal{F})$ such that $\pi(\omega, \cdot)=\delta_{\omega}$ on $\mathcal{F}_{0}$ for any $\omega \in \Omega$.

In analogy to these classical probabilistic notions, we now introduce the notions of a monetary risk kernel and of a regular conditional risk measure. Let us write $M_{i}=M_{b}\left(\Omega_{i}, \mathcal{F}_{i}\right)$ for $i=0,1$.

Definition 1.6. A monetary risk kernel from $\left(\Omega_{0}, \mathcal{F}_{0}\right)$ to $\left(\Omega_{1}, \mathcal{F}_{1}\right)$ is a real-valued function $\rho$ on $\Omega_{0} \otimes M_{1}$ such that

i) for each $\omega_{0} \in \Omega_{0}$, the functional $\rho\left(\omega_{0}, \cdot\right)$ is a monetary risk measure on $M_{1}$,

ii) for each $X \in M_{1}$, the function $\rho\left(\cdot, X_{1}\right)$ belongs to $M_{0}$.

Such a monetary risk kernel is called a convex risk kernel if each risk measure $\rho\left(\omega_{0}, \cdot\right)$ is convex.

Now consider a measurable space $(\Omega, \mathcal{F})$ and a sub- $\sigma$-field $\mathcal{F}_{0} \subseteq \mathcal{F}$.

Definition 1.7 A regular conditional risk measure on $(\Omega, \mathcal{F})$ with respect to $\mathcal{F}_{0}$ is defined as a monetary risk kernel $\rho_{0}$ from $\left(\Omega, \mathcal{F}_{0}\right)$ to $(\Omega, \mathcal{F})$ which is regular in the following sense:

$$
\rho_{0}\left(\omega, f\left(X_{0}, X\right)\right)=\rho_{0}\left(\omega, f\left(X_{0}(\omega), X\right)\right)
$$

for any $\omega \in \Omega$, any $X_{0} \in M_{0}$, any $X \in M$, and any bounded measurable function $f$ on $R^{2}$. 
Remark 1.8. Let $P$ be a probability measure on $(\Omega, \mathcal{F})$, and let $\rho_{0}$ be a regular conditional risk measure with respect to $\mathcal{F}_{0}$. Suppose that $\rho_{0}(\cdot, X)=\rho_{0}(\cdot, Y) P$-almost surely whenever $X=Y P$-almost surely. Then $\rho_{0}$ can also be regarded as a conditional monetary risk measure with respect to $\mathcal{F}_{0}$ in the sense of Definition 1.2. In view of the representation of conditional expectations by regular conditional distributions, it is natural to ask whether, conversely, any conditional risk measure can be represented by a regular conditional risk measure. This question is discussed in Section 2.2 of [T1].

\section{Consistency and law-invariance: a general result}

In this section we fix a probability space $(\Omega, \mathcal{F}, P)$ and a sub- $\sigma$-field $\mathcal{F}_{0}$ of $\mathcal{F}$. Let $\rho$ be a convex risk measure on $L^{\infty}$, and assume that $\rho$ is $\mathcal{F}_{0}$-consistent. Thus there is a conditional monetary risk measure $\rho_{0}$ with respect to $\mathcal{F}_{0}$ such that

$$
\rho=\rho\left(-\rho_{0}\right)
$$

Our aim is to show that $\mathcal{F}_{0}$-consistency together with law-invariance of $\rho$ implies that $\rho$ is an entropic risk measure. Note that we will also need strong sensitivity and the Lebesgue property of $\rho$ with respect to $P$, since these properties are necessary for $\rho$ to be entropic, as pointed out in Section 1.

Let us first collect some simple facts which will be used in our proof.

Lemma 2.1. If $\rho$ is strongly sensitive then $\rho_{0}$ is uniquely determined mod $P$.

Proof. Let $\rho_{0}$ and $\tilde{\rho}_{0}$ be two conditional risk measures such that $\rho=\rho\left(-\rho_{0}\right)=\rho\left(-\tilde{\rho}_{0}\right)$. Take $X \in L^{\infty}$; we want to show that $\rho_{0}(X)=\tilde{\rho}_{0}(X) P$-a.s.. Define $A_{0}=\left\{\rho_{0}(X)>\tilde{\rho}_{0}(X)\right\} \in \mathcal{F}_{0}$. Using the locality of $\rho_{0}$ we obtain

$$
\rho\left(X I_{A_{0}}\right)=\rho\left(-\rho_{0}\left(X I_{A_{0}}\right)\right)=\rho\left(-\rho_{0}(X) I_{A_{0}}\right),
$$

and the same equation holds for $\tilde{\rho}_{0}$. This implies

$$
\rho\left(-\rho_{0}(X) I_{A_{0}}\right)=\rho\left(-\tilde{\rho}_{0}(X) I_{A_{0}}\right),
$$

hence $P\left[A_{0}\right]=0$ due to the strong sensitivity of $\rho$. Thus we have $\rho_{0}(X) \leq \tilde{\rho}_{0}(X) P$-a.s., and the reverse inequality follows in the same manner.

The following lemma shows that law-invariance of the risk measure $\rho$ is inherited by the conditional risk measure $\rho_{0}$; its second part also appears in $[\mathrm{KS}]$ as Lemma 2.2.

Lemma 2.2. Let $\rho$ be strongly sensitive. Then law-invariance of $\rho$ implies conditional law-invariance of $\rho_{0}$, that is, $\rho_{0}(X)$ only depends on the conditional distribution of $X$ with respect to $\mathcal{F}_{0}$. In particular, we have $\rho_{0}(X)=\rho(X) P$-a.s. whenever $X$ is independent of $\mathcal{F}_{0}$.

Proof. Take $X$ and $Y$ in $L^{\infty}$ such that the conditional distributions $\mu_{X}\left(\cdot \mid \mathcal{F}_{0}\right)$ and $\mu_{Y}\left(\cdot \mid \mathcal{F}_{0}\right)$ coincide $P$-a.s. For any $A_{0} \in \mathcal{F}_{0}$, the random variables $X I_{A_{0}}$ and $Y I_{A_{0}}$ then have the same distribution, since

$$
E_{P}\left[f\left(X I_{A_{0}}\right)\right]=E\left[f(X) I_{A_{0}}+f(0) I_{A_{0}^{c}}\right]=E_{P}\left[\int f d \mu_{X}\left(\cdot \mid \mathcal{F}_{0}\right) I_{A_{0}}\right]+f(0) P\left[A_{0}^{c}\right]
$$

for any measurable function $f \geq 0$ on the real line. Law-invariance of $\rho$ implies $\rho\left(X I_{A_{0}}\right)=\rho\left(Y I_{A_{0}}\right)$ and hence, using $\rho=\rho\left(-\rho_{0}\right)$ and the locality of $\rho_{0}$,

$$
\rho\left(-\rho_{0}(X) I_{A_{0}}\right)=\rho\left(-\rho_{0}(Y) I_{A_{0}}\right)
$$

for any $A_{0} \in \mathcal{F}_{0}$. As in the proof of the preceding Lemma 2.1, it follows that $\rho_{0}(X)=\rho(X) P$-a.s., due to the strong sensitivity of $\rho$. 
If $X$ is independent of $\mathcal{F}_{0}$ with distribution $\mu$, then we have $\mu_{X}\left(\cdot \mid \mathcal{F}_{0}\right)=\mu P$-a.s., and so $\rho_{0}(X)$ is $P$ -a.s. constant. But this constant must be equal to $\rho(X)$, since cash-invariance and normalization of $\rho$ imply $\rho(X)=\rho\left(-\rho_{0}(X)\right)=\rho_{0}(X)$.

We are now going to show that $\mathcal{F}_{0}$-consistency together with law-invariance of $\rho$ implies that $\rho$ is entropic. In view of Remark 1.4, this will also require, in addition to the regularity properties mentioned above, some restriction on the $\sigma$-field $\mathcal{F}_{0}$ which ensures that we stay well away from the two extreme cases where the consistency condition becomes redundant.

Definition 2.3. Let us say that $\mathcal{F}_{0}$ is properly contained in $\mathcal{F}$ if

i) $\left(\Omega, \mathcal{F}_{0}, P\right)$ is atomless, and

ii) $(\Omega, \mathcal{F}, P)$ is conditionally atomless given $\mathcal{F}_{0}$, that is, there exists a uniformly distributed random variable $U$ on $(\Omega, \mathcal{F}, P)$ such that $U$ is independent of $\mathcal{F}_{0}$.

We are now ready to state our main result in this section:

Theorem 2.4. Assume that the convex risk measure $\rho$ is law-invariant, strongly sensitive, and satisfies the Lebesgue property. If $\rho$ is $\mathcal{F}_{0}$-consistent for some $\sigma$-field $\mathcal{F}_{0}$ which is properly contained in $\mathcal{F}$, then $\rho$ is an entropic risk measure with some parameter $\beta \in[0, \infty)$.

Proof. The proof is inspired by the characterization of certainty equivalents within the class of quasi-convex law-invariant risk measures in Lemma 11 of $[\mathrm{CMMM}]$. But our consistency assumption involves just one sub- $\sigma$-field $\mathcal{F}_{0}$ of $\mathcal{F}$, and this requires a conditional refinement of the argument in [CMMM]. We will proceed in two steps.

Consider the preference order $\prec$ on $L^{\infty}$ defined by

$$
X \prec Y: \Longleftrightarrow \rho(X)>\rho(Y)
$$

We write $X \preceq Y$ iff $\rho(X) \geq \rho(Y)$, and $X \sim Y$ iff $\rho(X)=\rho(Y)$. Since $(\Omega, \mathcal{F}, P)$ is atomless and $\rho$ is lawinvariant, this preference order can also be viewed as a preference order on lotteries, that is, on probability measures $\mu$ on the real line with bounded support:

$$
\mu \prec \nu: \Longleftrightarrow X \prec Y
$$

where $X$ and $Y$ are any random variables with distributions $\mu$ and $\nu$; for example, we could take $X=q_{\mu}(U)$ and $Y=q_{\nu}(U)$, where $q_{\mu}$ and $q_{\nu}$ are quantile functions of $\mu$, and where $\nu$ and $U$ is a random variable which is uniformly distributed on $(0,1)$. In the first step of the proof, we will show that this preference order on lotteries satisfies the axioms of von Neumann and Morgenstern and can thus be described in terms of expected utility for some strictly increasing continuous function $u$ on the real line.

Consider the functional $C$ on lotteries defined by

$$
C(\mu):=-\rho(X),
$$

and note that

$$
\mu \prec \nu \Longleftrightarrow C(\mu)<C(\nu) .
$$

In the second step of the proof, we will show that $C$ coincides with the certainty equivalent $C_{u}$ corresponding to $u$. But the certainty equivalent $C_{u}=C$ is also translation-invariant, due to the cash-invariance of $\rho$. This implies, by a classical argument of Bruno de Finetti, that $u$ must have a linear or an exponential form. Using the convexity of $\rho$, it will follow that $\rho$ is indeed an entropic risk measure.

Step 1. For constants $x>y$ we have $\rho(x)=-x<-y=\rho(y)$ by cash-invariance and normalization, hence $\delta_{x} \succ \delta_{y}$, and so the preference order on lotteries is monotone. It is also continuous with respect to the weak topology. More precisely, take any $c>0$, define $\mathcal{M}_{c}$ as the class of lotteries with support in $[-c, c]$, and let us show that the set $\left\{(\mu, \nu) \in \mathcal{M}_{c} \otimes \mathcal{M}_{c} \mid \mu \preceq \nu\right\}$ is weakly closed in $\mathcal{M}_{c} \otimes \mathcal{M}_{c}$. To this end we take lotteries 
$\mu_{n}$ and $\tilde{\mu}_{n}$ converging weakly to $\mu$ and $\tilde{\mu}$. Choose corresponding quantile functions $q_{n}, \tilde{q}_{n}, q, \tilde{q}$, and let $U$ be uniformly distributed on the unit interval $(0,1)$. Weak convergence implies almost sure convergence of $X_{n}:=q_{n}(U)$ to $X:=q(U)$ and of $\tilde{X}_{n}:=\tilde{q}_{n}(U)$ to $\tilde{X}:=\tilde{q}(U)$, and all these random variables are uniformly bounded by $c$. If $\mu_{n} \preceq \tilde{\mu}_{n}$ and hence $\rho\left(X_{n}\right) \geq \rho\left(\tilde{X}_{n}\right)$, the Lebesgue property of $\rho$ yields

$$
\rho(X)=\lim _{n \rightarrow \infty} \rho\left(X_{n}\right) \geq \lim _{n \rightarrow \infty} \rho\left(\tilde{X}_{n}\right)=\rho(\tilde{X}),
$$

and this implies $\mu \preceq \tilde{\mu}$.

Let us now verify the crucial independence axiom. Take lotteries $\mu, \nu, \gamma$ and any constant $\lambda \in(0,1)$. We have to show that $\mu \preceq \nu$ implies

$$
\lambda \mu+(1-\lambda) \gamma \preceq \lambda \nu+(1-\lambda) \gamma .
$$

Since $\mathcal{F}_{0}$ is properly contained in $\mathcal{F}$, we can find uniformly distributed random variables $U_{i}(i=0,1)$ such that $U_{0}$ is $\mathcal{F}_{0}$-measurable and $U_{1}$ is independent of $\mathcal{F}_{0}$. Define $X:=q_{\mu}\left(U_{1}\right), Y=q_{\nu}\left(U_{1}\right), Z=q_{\gamma}\left(U_{1}\right)$ and the set $A_{0}:=\left\{U_{0} \leq \lambda\right\} \in \mathcal{F}_{0}$. Thus we have $P\left[A_{0}\right]=\lambda$, and $X, Y$, and $Z$ are independent of $\mathcal{F}_{0}$ and have distributions $\mu, \nu$, and $\gamma$. Moreover, the random variable $X I_{A_{0}}+Z I_{A_{0}^{c}} \in L^{\infty}$ has distribution $\lambda \mu+(1-\lambda) \gamma$, since

$$
\begin{aligned}
E\left[f\left(X I_{A_{0}}+Z I_{A_{0}^{c}}\right)\right] & =E\left[f\left(X I_{A_{0}}+Z I_{A_{0}^{c}}\right) I_{A_{0}}\right]+E\left[f\left(X I_{A_{0}}+Z I_{A_{0}^{c}}\right) I_{A_{0}^{c}}\right] \\
& =E\left[f(X) I_{A_{0}}\right]+E\left[f(Z) I_{A_{0}^{c}}\right] \\
& =\lambda \int f d \mu+(1-\lambda) \int f d \gamma \\
& =\int f d(\lambda \mu+(1-\lambda) d \gamma)
\end{aligned}
$$

for any measurable function $f \geq 0$ on the real line. In the same way, $Y I_{A_{0}}+Z I_{A_{0}^{c}}$ has distribution $\lambda \nu+(1-\lambda) \gamma$. Now suppose that $\mu \preceq \nu$, hence $X \preceq Y$. In order to verify inequality (2.3), we show that

$$
\rho\left(X I_{A_{0}}+Z I_{A_{0}^{c}}\right) \geq \rho\left(Y I_{A_{0}}+Z I_{A_{0}^{c}}\right) .
$$

By Lemma 2.2, the independence of $X, Y$, and $Z$ of $\mathcal{F}_{0}$ implies $\rho_{0}(X)=\rho(X), \rho_{0}(Y)=\rho(Y)$, and $\rho_{0}(Z)=$ $\rho(Z) P$ - a.s.. Using the consistency condition (2.1) and the local property (1.1) of $\rho_{0}$, we obtain

$$
\begin{aligned}
\rho\left(X I_{A_{0}}+Z I_{A_{0}^{c}}\right) & =\rho\left(-\rho_{0}\left(X I_{A_{0}}+Z I_{A_{0}^{c}}\right)\right) \\
& =\rho\left(-\rho(X) I_{A_{0}}-\rho(Z) I_{A_{0}^{c}}\right) .
\end{aligned}
$$

But this implies inequality (2.4) since $\rho$ is monotone, and since our assumption $\mu \preceq \nu$ amounts to the inequality $-\rho(X) \leq-\rho(Y)$.

We have thus verified the axioms of von Neumann and Morgenstern which guarantee that the preference order $\prec$ on lotteries admits a numerical representation in terms of expected utility. More precisely, there exists a strictly increasing continuous function $u$ on the real line such that

$$
\mu \prec \nu: \Longleftrightarrow \int u d \mu<\int u d \nu ;
$$

see, for example, [FS, Corollary 2.28 and Proposition 2.33].

Step 2. Note that the functional $C$ on lotteries defined in $(2,2)$ coincides with the certainty equivalent $C_{u}$ associated to the function $u$, defined by

$$
C_{u}(\mu)=u^{-1}\left(\int u d \mu\right)
$$


Indeed, $u\left(C_{u}(\mu)\right)=\int u d \mu$ implies $\delta_{C_{u}(\mu)} \sim \mu$, hence

$$
C_{u}(\mu)=-\rho\left(C_{u}(\mu)\right)=-\rho(X)=C(\mu),
$$

where $X$ has distribution $\mu$, and where we have used cash-invariance and normalization of $\rho$ in the first equation. Thus our functional $C$ is indeed a certainty equivalent, namely equal to $C_{u}$. Moreover, the cash-invariance of $\rho$ implies that $C$ has the following translation property:

$$
C\left(T_{m} \mu\right)=-\rho(X+m)=-\rho(X)+m=C(\mu)+m
$$

for any constant $m$, where $T_{m} \mu$ denotes the shifted measure defined by $\int f d T_{m}(\mu)=\int f(x+m) \mu(d x)$. But a classical result of Bruno de Finetti, often called the Nagumo-Kolmogorov-de Finetti Theorem, says that a strictly increasing continuous function $u$, whose certainty equivalent has the translation property, must be either linear or of exponential form, that is, $u(x)=a+b x, u(x)=a-b e^{-\beta x}$, or $u(x)=a+b e^{\beta x}$ with strictly positive constants $b$ and $\beta$; see, for example, [FS Proposition 2.46] and the references therein. Note that so far we have only made use of the monetary properties of $\rho$, combined with law-invariance and $\mathcal{F}_{0}$-consistency. Now we use the convexity of $\rho$ to exclude the third case. But this shows that $\rho$ is an entropic risk measure $e_{\beta}$ with some parameter $\beta \geq 0$. More precisely, the first case implies that $\rho$ is linear, that is, $\rho(X)=E_{P}[-X]$, and in the second case $\rho$ is an entropic risk measure $e_{\beta}$ with parameter $\beta>0$.

Corollary 2.5. In the situation of Theorem 2.4, where $\rho$ is entropic with some parameter $\beta \in[0, \infty)$, also the conditional risk measure $\rho_{0}$ is entropic with the same parameter $\beta$, that is, we have $\rho_{0}(X)=e_{\beta, 0}(X)$ $P$-a.s. for any $X \in L^{\infty}$, where $e_{\beta, 0}$ is the conditional entropic risk measure defined in Remark 1.5.

Proof. By Theorem 2.4 we have $\rho=e_{\beta}$ for some $\beta \in[0, \infty)$. As pointed out in Remark 1.5, this implies $\rho\left(-e_{\beta, 0}\right)=\rho$, hence $\rho_{0}(X)=e_{\beta, 0}(X) P$ - almost surely for any $X \in L^{\infty}$, due to Lemma 2.1.

Remark 2.6. If the law-invariant risk measure $\rho$ in Theorem 2.5 is not only convex but even coherent then we obtain $\beta=0$, that is, $\rho$ reduces to the linear case $\rho(X)=E_{P}[-X]$. Even without the assumption of law-invariance, a coherent risk measure must be linear if the consistency assumption is required not just for one but for all sub- $\sigma$-fields $\mathcal{F}_{0}$ of $\mathcal{F}$; see $[\mathrm{Y}]$ and Theorem 6.3 in $[\mathrm{T}]$. Since the strong notion of consistency used in the standard literature and also in this paper has such drastic consequences and excludes many plausible risk measures, the question arises how it could be relaxed in a fruitful manner. For a discussion of this issue we refer to $[\mathrm{RS}],[\mathrm{T}],[\mathrm{T} 1],[\mathrm{FSv}]$ and the references therein.

\section{Local specification of random fields and of convex risk measures}

Let $I$ be a countable set of sites, and let $S$ be some polish state space with Borel $\sigma$-field $\mathcal{S}$. We assume that each site $i \in I$ can be in some state $s \in S$, and we denote by $\Omega=S^{I}$ the set of possible configurations $\omega: I \rightarrow S$. For any subset $J \subseteq I$, we denote by $\omega_{J}$ the restriction of $\omega$ to $J \subseteq I$, by $\mathcal{F}_{J}$ the $\sigma$-field on $\Omega$ generated by the projection maps $\omega \rightarrow \omega(i)$ for any $i \in J$, and we write $\mathcal{F}=\overline{\mathcal{F}}_{I}$. A probability measure $P$ on $(\Omega, \mathcal{F})$ will be called a random field.

Let $\mathcal{V}$ denote the class of non-empty finite subsets $V \subset I$. For a given set $V \in \mathcal{V}$, the $\sigma$-field $\mathcal{F}_{V}$ describes what is observable on $V$, while $\mathcal{F}_{V^{c}}$ describes the situation on $V^{c}:=I-V$, also called the environment of $V$.

Definition 3.1. A collection $\left(\pi_{V}\right)_{V \in \mathcal{V}}$ of regular conditional probability distributions $\pi_{V}$ on $(\Omega, \mathcal{F})$ with respect to $\mathcal{F}_{V^{c}}$ is called a local specification of a random field if it satisfies the consistency condition

$$
\pi_{W} \pi_{V}=\pi_{W}
$$

for any $V, W \in \mathcal{V}$ such that $V \subseteq W$. 
Note that each $\pi_{V}$ can be identified with a stochastic kernel $\tilde{\pi}_{V}$ from $\left(S^{V^{c}}, \mathcal{S}^{V^{c}}\right)$ to $\left(S^{V}, \mathcal{S}^{V}\right)$ such that $\pi_{V}(\omega, \cdot)=\delta_{\omega_{V}} \otimes \tilde{\pi}_{V}\left(\omega_{V^{c}}, \cdot\right)$. In the sequel we will freely use the same notation $\pi_{V}$ for both interpretations.

From now on we fix a local specification $\left(\pi_{V}\right)_{V \in \mathcal{V}}$ of a random field.

Definition 3.2. Let $\mathcal{P}$ denote the convex set of all random fields $P$ which are consistent with this local specification in the sense that

$$
P \pi_{V}=P \quad \text { for any } V \in \mathcal{V} .
$$

$A$ random field $P \in \mathcal{P}$ is also called a Gibbs measure. The case $|\mathcal{P}|>1$, where the global random field is not uniquely determined by the local specification $\left(\pi_{V}\right)_{V \in \mathcal{V}}$, is often referred to as a phase transition.

Note that, for any $V \in \mathcal{V}$ and any $P \in \mathcal{P}$, the stochastic kernel $\pi_{V}$ is a regular conditional probability distribution for $P$ with respect to the environment $\mathcal{F}_{V^{c}}$, and so we can write

$$
E_{P}\left[f \mid \mathcal{F}_{V^{c}}\right](\omega)=\int f(\eta) \pi_{V}(\omega, d \eta)
$$

for any measurable function $f \geq 0$ on $(\Omega, \mathcal{F})$.

In this probabilistic context three basic problems arise:

(I) A consistency problem: How arbitrarily can we prescribe the conditional probability distributions $\pi_{\{i\}}$ for single sites $i \in I$ such that they can be extended to a local specification $\left(\pi_{V}\right)_{V \in \mathcal{V}}$ in a consistent manner, that is, $\pi_{V} \pi_{\{i\}}=\pi_{V}$ for any $V \in \mathcal{V}$ and any $i \in V$ ?

(II) An existence problem: Is $\mathcal{P} \neq \emptyset$ ?

(III) A uniqueness and a representation problem: Is $|\mathcal{P}|=1$, and if not, does the convex set $\mathcal{P}$ admit a Choquet type representation in terms of its extreme points?

The answers are provided by the theory of Gibbs measures. As to Problem (I), a consistent extension is possible if the conditional distributions $\pi_{\{i\}}$ admit a Gibbsian representation in terms of some interaction potential, and such a representation is also necessary if the $\pi_{\{i\}}$ satisfy a mild condition of "quasi-locality"; see, for example, Section 2.3 in [G]. Problems (II) and (III) have a trivial answer if $I$ is finite. Indeed, in this case we have $I \in \mathcal{V}$ and $I^{c}=\emptyset$, and so the probability measure $P:=\pi_{I}(\omega, \cdot)$ does not depend on $\omega$. This implies $\mathcal{P}=\{P\}$, and so there is no phase transition. The situation becomes more involved in the infinite case $|I|=\infty$. In general, existence involves some tightness conditions. Here we simply assume it:

Assumption 3.3: The class $\mathcal{P}$ is not empty.

Let us now focus on the structure of the convex set $\mathcal{P}$ in the infinite case. To this end, we introduce the tail field

$$
\mathcal{F}_{\infty}:=\bigcap_{V \in \mathcal{V}} \mathcal{F}_{V^{c}}
$$

Using a method developed by E.B. Dynkin in the boundary theory of Markov processes, it can be shown that the local specification $\left(\pi_{V}\right)_{V \in \mathcal{V}}$ can be extended to a conditional probability distribution $\pi_{\infty}$ with respect to the tail field $\mathcal{F}_{\infty}$. More precisely, there exists a stochastic kernel $\pi_{\infty}$ from $\left(\Omega, \mathcal{F}_{\infty}\right)$ to $(\Omega, \mathcal{F})$ such that, for any $\omega \in \Omega$, the random field $\pi_{\infty}(\omega, \cdot)$ belongs to $\mathcal{P}$ and is actually an extreme point of the convex set $\mathcal{P}$; see $[\mathrm{Dy}],[\mathrm{Dy} 1]$, and $[\mathrm{F}]$. In particular we have

$$
\pi_{\infty} \pi_{V}=\pi_{\infty} \text { for any } V \in \mathcal{V}
$$

Moreover, $\pi_{\infty}$ provides, similtaneously for any $P \in \mathcal{P}$, a conditional distribution with respect to the tail field $\mathcal{F}_{\infty}$, that is,

$$
E_{P}\left[f \mid \mathcal{F}_{\infty}\right](\omega)=\int f(\eta) \pi_{\infty}(\omega, d \eta)
$$


$P$-a.s. for any $P \in \mathcal{P}$ and for any measurable function $f \geq 0$ on $(\Omega, \mathcal{F})$. Thus, any random field $P \in \mathcal{P}$ admits the representation

$$
P=\hat{P} \pi_{\infty}:=\int \pi_{\infty}(\omega, \cdot) \hat{P}(d \omega)
$$

where $\hat{P}$ denotes the restriction of $P$ to the $\sigma$-field $\hat{\mathcal{F}} \subseteq \mathcal{F}_{\infty}$ generated by the maps $\pi_{\infty}(\cdot, A)(A \in \mathcal{F})$. Conversely, any probability measure $\hat{P}$ on $(\Omega, \hat{\mathcal{F}})$ defines via $(3.4)$ a random field $P \in \mathcal{P}$. In this way, we obtain an integral representation of the convex set $\mathcal{P}$ that is coupled to the tail field by the kernel $\pi_{\infty}$ :

$$
\mathcal{P}=\left\{\hat{P} \pi_{\infty} \mid \hat{P} \text { is a probability measure on }(\Omega, \hat{\mathcal{F}})\right\} .
$$

In particular, a phase transition occurs if and only if the kernel $\pi_{\infty}$ does not depend on the tail field, that is, all measures $\pi_{\infty}(\omega, \cdot)$ coincide.

Remark 3.4. The integral representation (3.5) shows that the set of extreme points of the convex set $\mathcal{P}$ is given by $\mathcal{P}_{e}:=\left\{\pi_{\infty}(\omega, \cdot) \mid \omega \in \Omega\right\}$. Note that the map $\Phi: \Omega \rightarrow P_{e}$ given by $\omega \rightarrow \pi_{\infty}(\omega, \cdot)$ is measurable with respect to the $\sigma$-field $\hat{\mathcal{F}}$ on $\Omega$ and the canonical $\sigma$-field on $\mathcal{P}_{e}$ generated by the maps $P \rightarrow P[A](A \in \mathcal{F})$. Denoting by $\mu_{P}$ the image of $P$ under the map $\Phi$, the representation (3.4) takes the form

$$
P=\int_{\mathcal{P}_{e}} Q \mu_{P}(d Q) .
$$

Conversely, any probability measure $\mu$ on $\mathcal{P}_{e}$ defines via (3.6) a random field $P \in \mathcal{P}$, and we have $\mu=\mu_{P}$. Thus we obtain a Choquet type integral representation of the convex set $\mathcal{P}$, that is, any $P \in \mathcal{P}$ is barycenter of a unique probability measure $\mu_{P}$ on the set $\mathcal{P}_{e}$ of extreme points; see [Dy], [Dy1], and [F].

Let us now pass from the linear level of conditional probability distributions to the non-linear level of conditional risk measures.

Definition 3.5. A collection $\left(\rho_{V}\right)_{V \in \mathcal{V}}$ of convex risk kernels $\rho_{V}$ from $\left(\Omega, \mathcal{F}_{V^{c}}\right)$ to $(\Omega, \mathcal{F})$ is called a local specification of a convex risk measure if it satisfies the consistency condition

$$
\rho_{W}\left(-\rho_{V}\right)=\rho_{W}
$$

for any $V, W \in \mathcal{V}$ such that $V \subseteq W$, and if each kernel is regular in the sense of (1.7).

In analogy to the remark following Definition 3.1, each $\rho_{V}$ can be identified with a convex risk kernel $\tilde{\rho}_{V}$ from $\left(S^{V^{c}}, \mathcal{S}^{V^{c}}\right)$ to $\left(S^{V}, \mathcal{S}^{V}\right)$ such that $\rho_{V}(\omega, X)=\tilde{\rho}\left(\omega_{V^{c}}, X\left(\left(\omega_{V^{c}}, \cdot\right)\right)\right)$. Here again, we will freely use the same notation $\rho_{V}$ for both interpretations.

From now on we fix a local specification $\left(\rho_{V}\right)_{V \in \mathcal{V}}$ of a convex risk measure.

Definition 3.6. Let $\mathcal{R}$ denote the set of all convex risk measures $\rho$ on $M$ which are consistent with the local specification $\left(\rho_{V}\right)_{V \in \mathcal{V}}$, that is,

$$
\rho\left(-\rho_{V}\right)=\rho \quad \text { for any } V \in \mathcal{V} \text {. }
$$

For the local specification $\left(\rho_{V}\right)_{V \in \mathcal{V}}$ and for the corresponding class $\mathcal{R}$ of global risk measures, we can now formulate, as we did already in the Introduction, a consistency problem analogous to (I), an existence problem analogous to (II), and a uniqueness and representation problem analogous to (III). The consistency problem is open. For the analogues of Problems (II) and (III), some partial results are discussed in [CFK]. In the next section we will show that all three problems have an explicit solution if we introduce an additional condition of local law invariance, combined with some further regularity conditions. 


\section{The case of local law-invariance}

Let us formulate a condition of law-invariance for our local specification $\left(\rho_{V}\right)_{V \in \mathcal{V}}$ of a convex risk measure with respect to the local specification $\left(\pi_{V}\right)_{V \in \mathcal{V}}$ of a random field. Note that it amounts to a condition of local law-invariance for the risk measures in the class $\mathcal{R}$.

Definition 4.1. We say that $\left(\rho_{V}\right)_{V \in \mathcal{V}}$ is law-invariant with respect to $\left(\pi_{V}\right)_{V \in \mathcal{V}}$ if, for any $\omega \in \Omega$ and any $V \in \mathcal{V}$, the convex risk measure $\rho_{V}(\omega, \cdot)$ is law-invariant with respect to the probability measure $\pi_{V}(\omega, \cdot)$. In the same way, we say that $\left(\rho_{V}\right)_{V \in \mathcal{V}}$ has the Lebesgue property with respect to $\left(\pi_{V}\right)_{V \in \mathcal{V}}$ if any risk measure $\rho_{V}(\omega, \cdot)$ has the Lebesgue property with respect to $\pi_{V}(\omega, \cdot)$. Strong sensitivity of $\left(\rho_{V}\right)_{V \in \mathcal{V}}$ with respect to $\left(\pi_{V}\right)_{V \in \mathcal{V}}$ is defined in the same manner.

As an example, let us consider the entropic case. We use the notation $e_{V, \beta}(\omega, \cdot)$ for the entropic risk measure defined by

$$
e_{V, \beta}(\omega, X)=\frac{1}{\beta} \log \int e^{-\beta X(\eta)} \pi_{V}(\omega, d \eta)
$$

for any parameter $\beta \in[0, \infty)$; for $\beta=0$, the right-hand side should be read as $\int(-X)(\eta) \pi_{V}(\omega, d \eta)$, as in Remark 1.5. Now let $\beta_{V}(\cdot)$ be an $\mathcal{F}_{V^{c}}$-measurable function with values in $[0, \infty)$, and consider the convex risk kernel defined by

$$
\rho_{V}(\omega, \cdot)=e_{V, \beta_{V}(\omega)}(\omega, \cdot) .
$$

Note that each risk measure $\rho_{V}(\omega, \cdot)$ is clearly law-invariant, strongly sensitive, and has the Lebesgue property with respect to the probability measure $\pi_{V}(\omega, \cdot)$. Moreover, each risk kernel $\rho_{V}$ is regular in the sense of Definition (1.7).

The question remains whether the entropic risk kernels $\rho_{V}$ defined by (4.2) for any $V \in \mathcal{V}$ satisfy the consistency condition (3.7) of a local specification. This is clearly the case if all functions $\beta_{V}$ are constant and coincide with the same parameter $\beta \in[0, \infty)$. In order to formulate a condition which is both sufficient and necessary, let us first slightly extend the definition of a local specification.

Definition 4.2. We say that a property holds modulo $\mathcal{P}$ if it holds $P$-almost surely for any $P \in \mathcal{P}$. Let us also say that a family $\left(\rho_{V}\right)_{V \in \mathcal{V}}$ of convex risk kernels forms a local specification modulo $\mathcal{P}$ if the consistency condition

$$
\rho_{W}\left(-\rho_{V}\right)(\omega, \cdot)=\rho_{W}(\omega, \cdot) \quad \text { for any } V, W \in \mathcal{V} \text { such that } V \subseteq W
$$

is satisfied modulo $\mathcal{P}$.

Lemma 4.3. The family of entropic risk kernels $\left(\rho_{V}\right)_{V \in \mathcal{V}}$ defined by (4.2) forms a local specification modulo $\mathcal{P}$ if and only if there is an $\mathcal{F}_{\infty}$-measurable function $\beta_{\infty}$ with values in $[0, \infty)$ such that the equality

$$
\beta_{V}=\beta_{\infty} \quad \text { for any } V \in \mathcal{V}
$$

holds modulo $\mathcal{P}$.

\section{Proof.}

1) To show "if", let $\beta_{\infty}$ be an $\mathcal{F}_{\infty}$-measurable function with values in $[0, \infty)$, and consider the entropic risk kernels $\tilde{\rho}_{V}$ defined by $\left(\tilde{\rho}_{V}\right)(\omega, \cdot)=e_{V, \beta_{\infty}(\omega)}(\omega, \cdot)$. This family satisfies the consistency condition $\left(\tilde{\rho}_{V}\right)\left(-\tilde{\rho}_{W}\right)=$ $\tilde{\rho}_{V}$ for $V \subseteq W$ and is thus a local specification of a convex risk measure. Now note that condition (4.4) implies that the equality

$$
\tilde{\rho}_{V}(\omega, \cdot)=\rho_{V}(\omega, \cdot) \quad \text { for any } V \in \mathcal{V}
$$

holds modulo $\mathcal{P}$, and this implies that the consistency condition (4.3) for $\left(\rho_{V}\right)_{V \in \mathcal{V}}$ is satisfied modulo $\mathcal{P}$. 
2) To show "only if", assume that condition (4.3) holds for $\omega \in \Omega$. Since $\rho_{W}(\omega, \cdot)$ is entropic with parameter $\beta_{W}(\omega)$, we also have

$$
\rho_{W}\left(-e_{V, \beta_{W}(\omega)}\right)(\omega, \cdot)=\rho_{W}(\omega, \cdot) .
$$

By Lemma 2.1, this implies

$$
\rho_{V}(\eta, X)=e_{V, \beta_{W}(\omega)}(\eta, \cdot) \quad \pi_{W}(\omega, \cdot)-\text { a.s.. }
$$

Since both sides are entropic with parameters $\beta_{V}(\eta)$ and $\beta_{W}(\omega)$, respectively, the argument in part 2) of the proof of Theorem 4.5 shows that

$$
\beta_{V}(\cdot)=\beta_{W}(\omega) \quad \pi_{W}(\omega, \cdot)-\text { a.s.. }
$$

For any $W \in \mathcal{V}$, this implies that the equality

$$
\beta_{V}=\beta_{W} \quad \text { for any } V \in \mathcal{V}
$$

holds modulo $\mathcal{P}$. Now fix a sequence $\left(V_{n}\right) \subseteq \mathcal{V}$ increasing to $I$. The function $\beta_{\infty}$ defined by

$$
\beta_{\infty}(\omega):=I_{A}(\omega) \lim \sup \beta_{V_{n}}(\omega)
$$

with $A:=\left\{\lim \sup _{n} \beta_{V_{n}}<\infty\right\}$ is clearly $\mathcal{F}_{\infty}$-measurable and takes values in $[0, \infty)$. For any $P \in \mathcal{P}$ we have $\beta_{V_{n}}=\beta_{V_{n+1}}(n=1,2, \ldots) P$-a.s., hence $\beta_{\infty}=\beta_{V_{n}}(n=1,2, \ldots) P$-a.s.. In view of $(4.5)$, this implies that the equations

$$
\beta_{\infty}=\beta_{V} \quad \text { for any } V \in \mathcal{V}
$$

hold modulo $\mathcal{P}$, and hence the same is true for the equations

$$
\rho_{V}(\omega, \cdot)=e_{V, \beta_{\infty}(\omega)}(\omega, \cdot) \quad \text { for any } V \in \mathcal{V}
$$

Since the family $\left(e_{V, \beta_{\infty}}\right)_{V \in \mathcal{V}}$ is a local specification, it follows that the family $\left(\rho_{V}\right)_{V \in \mathcal{V}}$ is a local specification modulo $\mathcal{P}$.

An entropic local specification is clearly law-invariant, strongly sensitive, and has the Lebesgue property with respect to $\left(\pi_{V}\right)_{V \in \mathcal{V}}$. Our aim is to show that also the converse holds. To this end, we introduce the following equivalence assumption on the underlying local specification $\left(\pi_{V}\right)_{V \in \mathcal{V}}$ of a random field. Note that it implies local equivalence of the random fields in $\mathcal{P}$, that is, any two random fields in $\mathcal{P}$ are equivalent on the $\sigma$-field $\mathcal{F}_{V}$ for any $V \in \mathcal{V}$.

Assumption 4.4. For any $V \in \mathcal{V}$, the measures $\pi_{V}(\omega, \cdot)$ are all atomless and equivalent on the $\sigma$-field $\mathcal{F}_{V}$.

We are now ready to prove the following converse result.

Theorem 4.5 If the local specification $\left(\rho_{V}\right)_{V \in \mathcal{V}}$ of a convex risk measure is law-invariant, strongly sensitive and has the Lebesgue property with respect to $\left(\pi_{V}\right)_{V \in \mathcal{V}}$, then it is entropic. More precisely, there exists an $\mathcal{F}_{\infty}$-measurable function $\beta_{\infty}$ with values in $[0, \infty)$ such that we have, modulo $\mathcal{P}$,

$$
\rho_{V}(\omega, X)=\frac{1}{\beta_{\infty}(\omega)} \log \int e^{-\beta_{\infty}(\omega) X(\eta)} \pi_{V}(\omega, d \eta)
$$

for any $V \in \mathcal{V}$ and any $X \in M$.

Proof. 1) Fix $\omega \in \Omega$ and $V \in \mathcal{V}$ with $|V|>1$. For any $i \in V$, assumption 4.4 implies that the $\sigma$-field $\mathcal{F}_{V-\{i\}}$ is properly contained in $\mathcal{F}_{V}$ with respect to the probability measure $\pi_{V}(\omega, \cdot)$. Now consider the convex risk measure $\rho_{V}(\omega, \cdot)$ on $L^{\infty}\left(\Omega, \mathcal{F}_{V}, \pi_{V}(\omega, \cdot)\right)$. It is law-invariant, strongly sensitive, and has the Lebesgue property with respect to $\pi_{V}(\omega, \cdot)$ on $\mathcal{F}_{V}$. Moreover, it is $\mathcal{F}_{V-\{i\}}$-consistent, since $\rho_{V}\left(-\rho_{\{i\}}\right)(\omega, \cdot)=\rho_{V}(\omega, \cdot)$. By Theorem 2.1 it follows that $\rho_{V}(\omega, \cdot)$ is entropic, that is, equation (4.2) holds for some parameter $\beta_{V}(\omega) \in$ $[0, \infty)$. 
2) Since $\rho_{V}$ is a convex risk kernel, the function $\rho_{V}(\cdot, X)$ is $\mathcal{F}_{V^{c}}$ measurable for any $X \in M$, and this implies that the same is true for the parameter $\beta_{V}(\cdot)$ appearing in part 1). Indeed, for $A \in \mathcal{F}_{V}$ such that $P[A] \in(0,1)$, we have $\pi_{V}(\omega, A) \in(0.1)$ due to Assumption 4.4, and so the map

$$
\beta \rightarrow \rho\left(\omega,-I_{A}\right)=\frac{1}{\beta} \log \left(1+\left(e^{\beta}-1\right) \pi_{V}(\omega, A)\right)
$$

is smooth and strictly increasing in $\beta$. Inverting this map, we obtain $\beta_{V}(\omega)$ as a smooth function of $\rho\left(\omega,-I_{A}\right)$,

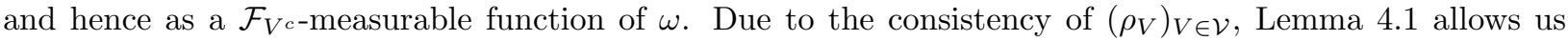
to conclude that there exists an $\mathcal{F}_{\infty}$-measurable function $\beta_{\infty}$ with values in $[0, \infty)$ such that the equation $\beta_{\infty}(\omega)=\beta_{V}(\omega)$ for any $V \in \mathcal{V}$ holds modulo $\mathcal{P}$. But this means that equation (4.2) holds modulo $\mathcal{P}$.

3) It remains to show that the entropic representation (4.6) also holds for the case $|V|=1$, that is, for the single sites $i \in I$. Take $V \in \mathcal{V}$ such that $i \in V$ and $|V|>1$. Then, $P$-almost surely for any $P \in \mathcal{P}$, the risk measure $\rho_{V}(\omega, \cdot)$ has the entropic form (4.6) with parameter $\beta_{\infty}(\omega)$. A direct computation shows that $\rho_{V}\left(-e_{\{i\}, \beta_{\infty}}\right)(\omega, \cdot)=\rho_{V}(\omega, \cdot)$. But we also have $\rho_{V}\left(-\rho_{\{i\}}\right)(\omega, \cdot)=\rho_{V}(\omega, \cdot)$, due to consistency. By Lemma (2.1), this yields $\rho_{\{i\}}(\eta, \cdot)=e_{\{i\}, \beta_{\infty}}(\eta, \cdot)$ for $\pi_{V}(\omega, \cdot)$ - almost all $\eta$, and hence modulo $\mathcal{P}$.

In the locally law-invariant case, we can now state the solution of the consistency problem for spatial risk measures, formulated in the introduction in analogy to Problem (I) in Section 3: How arbitrarily can we prescribe the conditional risk measures at the single sites in $I$ so that they admit a consistent extension to a local specification? The answer is that they must have an entropic form:

Corollary 4.6. Suppose that at each site $i \in I$, we have specified a regular convex risk kernel $\rho_{\{i\}}$ from $\left(\Omega, \mathcal{F}_{\{i\}^{c}}\right)$ to $(\Omega, \mathcal{F})$ such that each risk measure $\rho_{\{i\}}(\omega, \cdot)$ is law-invariant with respect to the probability measure $\pi_{\{i\}}(\omega, \cdot)$. The collection $\left(\rho_{\{i\}}\right)_{i \in I}$ can be extended to a local specification $\left(\rho_{V}\right)_{V \in \mathcal{V}}$ modulo $\mathcal{P}$ that is law-invariant with respect to $\left(\pi_{V}\right)_{V \in \mathcal{V}}$ if and only if, modulo $\mathcal{P}$, the risk kernels $\rho_{\{i\}}(\omega, \cdot)$ are all entropic with the same tail-measurable parameter $\beta_{\infty}(\omega)$, that is,

$$
\rho_{\{i\}}(\omega, X)=\frac{1}{\beta_{\infty}(\omega)} \log \int e^{-\beta_{\infty}(\omega) X(\eta)} \pi_{\{i\}}(\omega, d \eta)
$$

for any $X \in M$.

Let us now fix an entropic local specification $\left(\rho_{V}\right)_{V \in \mathcal{V}}$ of the form (4.6), and let us turn to the analogues of the existence problem (II) and the representation problem (III) in Section 3. The answers are immediate if the set $I$ is finite. Indeed, in this case the probability measure $\pi_{I}(\omega, \cdot)$ does not depend on $\omega$, and there is exactly one random field in $\mathcal{P}$, namely $P=\pi_{I}$. Moreover, the tail field is trivial, the parameter $\beta_{\infty}$ is constant, and so there is exactly one risk measure in the class $\mathcal{R}$, namely the entropic risk measure $\rho_{I}$ defined in terms of $\pi_{I}$ and $\beta_{\infty}$.

From now on we focus on the case $|I|=\infty$ where the situation is more involved. As in the case of Gibbs measures, we need a limit procedure in order to clarify the structure of the global risk measures consistent with the local specification $\left(\rho_{V}\right)_{V \in \mathcal{V}}$. Let $\mathcal{R}_{L}$ denote the class of all risk measures $\rho \in \mathcal{R}$ that have the Lebesgue property with respect to the class $\mathcal{P}$. By this we mean that $\rho(X)=\lim _{n \rightarrow \infty} \rho\left(X_{n}\right)$ whenever $\left(X_{n}\right)$ is a uniformly bounded sequence in $M$ such that $\lim _{n \rightarrow \infty} X_{n}=X$ modulo $\mathcal{P}$. Our aim is to describe the structure of the set $\mathcal{R}_{L}$ in more detail. To this end, we first extend the local specification $\left(\rho_{V}\right)_{V \in \mathcal{V}}$ defined by (4.6) to the tail field $\mathcal{F}_{\infty}$ by defining the entropic risk kernel

$$
\rho_{\infty}(\omega, X)=\frac{1}{\beta_{\infty}(\omega)} \log \int e^{-\beta_{\infty}(\omega) X(\eta)} \pi_{\infty}(\omega, d \eta)
$$

from $\left(\Omega, \mathcal{F}_{\infty}\right)$ to $(\Omega, \mathcal{F})$; recall that $\pi_{\infty}(\omega, d \eta)$ is the conditional distribution with respect to the tail field associated to the local specification $\left(\pi_{V}\right)_{V \in \mathcal{V}}$ via $(3.4)$. Note that $\rho_{\infty}$ is actually a convex risk kernel from $(\Omega, \hat{\mathcal{F}})$ to $(\Omega, \mathcal{F})$, where $\hat{\mathcal{F}}$ is the sub- $\sigma$-field of $\mathcal{F}_{\infty}$ appearing in $(3.5)$. 
We denote by $\hat{M}:=M_{b}(\Omega, \hat{\mathcal{F}})$ the space of all bounded measurable functions on $(\Omega, \hat{\mathcal{F}})$, and by $\hat{\mathcal{R}}_{L}$ the class of all convex risk measures on $\hat{M}$ which have the Lebesgue property with respect to the class $\mathcal{P}$. The following representation (4.8) of the class $\mathcal{R}_{L}$ can be seen as a non-linear analogue to the integral representation (3.5) of the class $\mathcal{P}$ of Gibbs measures.

Theorem 4.7 For any $\omega \in \Omega$, the risk measure $\rho_{\infty}(\omega, \cdot)$ belongs to $\mathcal{R}_{L}$, and so we have

$$
\rho_{\infty}\left(-\rho_{V}\right)=\rho_{\infty} \quad \text { for any } V \in \mathcal{V} \text {. }
$$

In particular the set $\mathcal{R}_{L}$ is not empty, and it has the form

$$
\mathcal{R}_{L}=\left\{\hat{\rho}\left(-\rho_{\infty}\right) \mid \hat{\rho} \in \hat{\mathcal{R}}_{L}\right\}
$$

Proof. 1) To see that $\rho_{\infty}(\omega, \cdot) \in \mathcal{R}_{L}$, note first that $\rho_{\infty}(\omega, \cdot)$ has the Lebesgue property with respect to $\mathcal{P}$, since $\pi_{\infty}(\omega, \cdot) \in \mathcal{P}$. Moreover, $\pi_{\infty}(\omega, \cdot) \in \mathcal{P}$ implies

$$
\rho_{V}(\omega, \cdot)=e_{V, \beta_{\infty}}(\omega, \cdot) \quad \pi_{\infty}(\omega, \cdot) \text {-almost surely, }
$$

hence $\rho_{\infty}\left(-\rho_{V}\right)(\omega, \cdot)=\rho_{\infty}(\omega, \cdot)$ for any $V \in \mathcal{V}$, and so we have $\rho_{\infty}(\omega, \cdot) \in \mathcal{R}$.

2) In order to prove the inclusion $\subseteq$ in equation (4.8), take $\rho \in \mathcal{R}_{L}$ and define $\hat{\rho}$ as the restriction of $\rho$ to $\hat{M}$. Clearly we have $\hat{\rho} \in \hat{\mathcal{R}}_{L}$. Take $X \in M$; we have to show $\rho(X)=\hat{\rho}\left(-\rho_{\infty}(\cdot, X)\right)$. To this end, we fix a sequence $\left(V_{n}\right)_{n=1,2, \ldots} \subset \mathcal{V}$ which increases to $I$. Note first that we have

$$
\begin{aligned}
\rho_{\infty}(\cdot, X) & =\frac{1}{\beta_{\infty}} \log E_{P}\left[e^{-\beta_{\infty} X} \mid \mathcal{F}_{\infty}\right] \\
& =\lim _{n \rightarrow \infty} \frac{1}{\beta_{\infty}} \log E_{P}\left[e^{-\beta_{\infty} X} \mid \mathcal{F}_{V_{n}^{c}}\right] \\
& =\lim _{n \rightarrow \infty} \rho_{V_{n}}(\cdot, X)
\end{aligned}
$$

$P$-a.s. for all $P \in \mathcal{P}$, due to backward martingale convergence. Using the consistency condition $\rho=\rho\left(-\rho_{V_{n}}\right)$ and the Lebesgue property of $\rho$ with respect to $\mathcal{P}$, we obtain

$$
\begin{aligned}
\rho(X) & =\lim _{n \rightarrow \infty} \rho\left(-\rho_{V_{n}}(X)\right) \\
& =\rho\left(-\rho_{\infty}(X)\right) \\
& =\hat{\rho}\left(-\rho_{\infty}(X)\right) .
\end{aligned}
$$

3) In order to show the reverse inclusion $\supseteq$, take $\hat{\rho} \in \hat{\mathcal{R}}_{L}$ and define $\rho:=\hat{\rho}\left(-\rho_{\infty}\right)$. Then we have $\rho \in \mathcal{R}$, since

$$
\rho\left(-\rho_{V}\right)=\hat{\rho}\left(-\rho_{\infty}\left(-\rho_{V}\right)\right)=\hat{\rho}\left(-\rho_{\infty}\right)=\rho
$$

for any $V \in \mathcal{V}$. Moreover, $\rho$ has the Lebesgue property with respect to $\mathcal{P}$. Indeed, if $\left(X_{n}\right)_{n=1,2, \ldots}$ is a uniformly bounded sequence in $M$ such that $X_{n} \rightarrow X P$-a.s. for any $P \in \mathcal{P}$, then we obtain, using the Lebesgue property first for $\rho_{\infty}$ and then for $\hat{\rho}$,

$$
\begin{aligned}
\rho(X) & =\hat{\rho}\left(-\rho_{\infty}(X)\right) \\
& =\hat{\rho}\left(-\lim _{n \rightarrow \infty} \rho_{\infty}\left(X_{n}\right)\right) \\
& =\lim _{n \rightarrow \infty} \hat{\rho}\left(-\rho_{\infty}\left(X_{n}\right)\right) \\
& =\lim _{n \rightarrow \infty} \rho\left(X_{n}\right) .
\end{aligned}
$$

The representation (4.8) shows that we have $\left|\mathcal{R}_{L}\right|=1$ if and only if the risk measures $\rho_{\infty}(\omega, \cdot)$ in $(4.7)$ all coincide. Thus there are two possible sources of multiplicity for the global risk measures in the class $\mathcal{R}_{L}$. 
The first source is a probabilistic phase transition, that is, a multiplicity in the class $\mathcal{P}$ of global random fields, since this amounts to non-uniqueness of the measure $\pi_{\infty}(\omega, \cdot)$ in $(3.4)$. The second source is the tail dependence of the parameter $\beta_{\infty}$. To see the separate effect of these two sources more clearly, let us state the following two corollaries.

Corollary 4.8. Let us assume that the parameter $\beta_{\infty}$ is constant. Then the local specification $\left(\rho_{V}\right)_{V \in \mathcal{V}}$ determines a unique global risk measure $\rho \in \mathcal{R}_{L}$ if and only if there is no probabilistic phase transition, that $i s$, iff $|\mathcal{P}|=1$. In that case, $\rho$ coincides with the entropic risk measure $e_{\beta_{\infty}}$ defined by the unique random field $P \in \mathcal{P}$ and the constant parameter $\beta_{\infty}$.

Proof. If $\mathcal{P}$ contains only one random field $P$, then the measures $\pi_{\infty}(\omega, \cdot)$ in $(3.4)$ are all equal to $P$. This implies that the risk measure $\rho_{\infty}(\omega, \cdot)$ does not depend on $\omega$, since (4.7) reduces to

$$
\rho_{\infty}(\omega, X)=\frac{1}{\beta_{\infty}} \log \int e^{-\beta_{\infty} X} d P
$$

and since $\beta_{\infty}$ is assumed to be constant. This means that $\rho_{\infty}(\omega, \cdot)$ coincides with the unconditional entropic risk measure $e_{\beta_{\infty}}$ defined in terms of $P$. Conversely, a probabilistic phase transition means that $\pi_{\infty}(\omega, \cdot) \neq$ $\pi_{\infty}\left(\omega_{1}\right)$ for some $\omega_{1}, \omega_{2} \in \Omega$, and this implies $\rho_{\infty}\left(\omega_{1}, \cdot\right) \neq \rho_{\infty}\left(\omega_{2}, \cdot\right)$, hence $\left|\mathcal{R}_{L}\right|>1$.

Corollary 4.9. Suppose that $|\mathcal{P}|=1$. Then we have $\left|\mathcal{R}_{L}\right|=1$ if and only if $\beta_{\infty}$ is constant.

Proof. The implication "if" follows from the preceding Corollary. Conversely, assume that $\mathcal{P}=\{P\}$ and $\left|\mathcal{R}_{L}\right|=1$. Then the risk measure in (4.7) takes the form

$$
\rho_{\infty}(\omega, X)=\frac{1}{\beta_{\infty}(\omega)} \log \int e^{-\beta_{\infty}(\omega) X} d P
$$

For any $X \in M$ which is not constant $P$-almost surely, the function $\beta \rightarrow \log E_{P}\left[e^{-\beta X}\right]$ is strictly convex. This implies that the right hand side of (4.8) is strictly monotone in the parameter $\beta_{\infty}(\omega)$. But the left hand side does not depend on $\omega$ since $\left|\mathcal{R}_{L}\right|=1$, and so $\beta_{\infty}(\omega)$ must be constant.

In the preceding discussion, law-invariance was only assumed in a local sense, namely for the local specification $\left(\rho_{V}\right)_{V \in \mathcal{V}}$. The assumption of global law-invariance of a spatial risk measure with respect to some random field $P$ would be much more restrictive, as the following corollary shows.

Corollary 4.10. Suppose that $\rho$ is a risk measure in $\mathcal{R}_{L}$ that is law-invariant and strongly sensitive with respect to some random field $P$. Then we have $P \in \mathcal{P}$, there is a constant $\beta_{P} \in[0, \infty)$ such that $\beta_{\infty}(\cdot)=\beta_{P}$ $P$-almost surely, and $\rho$ is the entropic risk measure defined in terms of $P$ and $\beta_{P}$.

Proof. Take any $V \in \mathcal{V}$. Due to Theorem (2.4), the consistency condition $\rho=\rho\left(-\rho_{V}\right)$ together with law-invariance and the assumed regularity properties of $\rho$ with respect to $P$ imply that $\rho$ is an entropic risk measure, defined in terms of $P$ and some parameter $\beta_{P} \in[0, \infty)$. Since $\rho=\rho\left(-e_{V}\right)$, where $e_{V}(\omega, \cdot)$ denotes the entropic risk measure defined by $\pi_{V}(\omega, \cdot)$ and the parameter $\beta_{P}$, we obtain $\rho_{V}(\cdot, X)=e_{V}(\cdot, X) P$-almost surely for any $X \in M$, due to Lemma 2.1. But this implies that $\beta_{P}=\beta_{\infty}(\cdot) P$-almost surely, and that $\pi_{V}$ is a conditional probability distribution for $P$ with respect to $\mathcal{F}_{V^{c}}$. Since $V \in \mathcal{V}$ was arbitrary, we have shown $P \in \mathcal{P}$.

Acknowledgements. In part, the results of this paper were obtained while the author was visiting the FIM at ETH Zürich, the Departments of Mathematics at TU and LMU München and the National University of Singapore, and the Hausdorff Institute of Mathematics at the University of Bonn. It is a pleasure to express my thanks for their support. 


\section{References}

[ADEH] Artzner, P., Delbaen, F., Eber, J.-M., and Heath, D., Coherent measures of risk, Mathematical Finance, 9, 203 - 228 (1999)

[CFK] Cotar, C., Föllmer, H., and Klüppelberg, C.: Spatial risk measures. Work in Progress.

[CK] Cheridito, P. and Kupper, M., Composition of time-consistent dynamic monetary risk measures in discrete time, Int. J. Theor. Appl. Finance, 14, 137 - 162 (2011)

[CMMM] Cerreia-Vioglio, S., Maccheroni, F., Marinacci, M., and Montrucchio, L., Risk measures: rationality and diversification, Mathematical Finance, 21, 743 - 774 (2011)

[D] Dobrushin, R. L.: Description of a random field by means of conditional probabilities and conditions of its regularity, Theor. Probability Appl., 13, 197 - 224 (1968)

[DS] Detlefsen, K. and Scandolo, G., Conditional and dynamic convex risk measures, Finance and Stochastics, $9 / 4,539-561(2005)$

[Dy] Dynkin, E. B., Entrance and Exit Spaces for a Markov Process. Actes Congrès Intern. Math., vol. 2, $507-512(1971)$

[Dy1] Dynkin, E. B., Integral Representation of Excessive Measures and Excessive Functions. Russ. Math. Surveys 27, 43 - 84 (1972)

[FSv] Fasen, V. and Svejda, A., Time Consistency of Multi-Period Distortion Measures, Statistics \& Risk Modeling 29, 133-153 (2012)

[F] Föllmer, H., Phase transition and Martin boundary, Séminaire de Probabilités (Strasbourg) 9, 305 - 317 (1975)

[FK] Föllmer, H. and Knispel, Th., Convex Risk Measures: Basic Facts, Law-invariance and beyond, Asymptotics for Large Portfolios. To appear in: Handbook of the Fundamentals of Financial Decision Making, Eds. L.C. MacLean and W.T. Ziemba, World Scientific (2013)

[FS] Föllmer, H. and Schied, A., Stochastic finance-An introduction in discrete time, 3rd edition, Graduate Textbook Series, Walter de Gruyter \& Co., Berlin, (2011)

[FS1] Föllmer, H. and Schied, A., Convex measures of risk and trading constraints, Finance and Stochastics, 6/4, $429-447(2002)$

[FRG] Frittelli, M. and Rosazza Gianin, E., Putting order in risk measures, Journal of Banking and Finance, $26,1473-1486(2002)$

[G] Georgii, H.-O., Gibbs measures and Phase Transitions, 2nd ext. edition, De Gruyter Studies in Mathematics 9 (2011)

[H] Heath, D., Back to the future, Plenary Lecture at the First World Congress of the Bachelier Society, Paris (2000)

[KS] Kupper, M. and Schachermayer, W., Representation results for law invariant time consistent functions, Math. Financ. Econ., 2/3, 189 - 210 (2009)

[RS] Roorda, B. and Schumacher, J.M., Time consistency conditions for acceptability measures - with an application to Tail Value at Risk, Insurance: Mathematics and Economics 40/2, 209-230 (2007)

[T] Tutsch, S., Update rules for convex risk measures, Quantitative Finance, 8/8, 833 - 843 (2008)

[T1] Tutsch, S., Konsistente und konsequente dynamische Risikomaße und das Problem der Aktualisierung, PhD thesis, Humboldt-Universität zu Berlin (2006) 
[Y] Yoo, K.-R., The iterative law of expectation and non-additive probability measures, Economics Letters 37, 145 - 149 (1991) 